\title{
EL CAMINO A LA ELIMINACIÓN: UN PANORAMA DE LAS ENFERMEDADES INFECCIOSAS DESATENDIDAS EN AMÉRICA LATINA Y EL CARIBE
}

\author{
THE ROADTO ELIMINATION: AN OVERVIEW OF NEGLECTED INFECTIOUS DISEASES IN \\ LATIN AMERICA AND THE CARIBBEAN
}

\author{
Steven Kenyon Ault ${ }^{1, a}$, Laura Catalá Pascual ${ }^{1, b}$, Maria Elena Grados-Zavala ${ }^{1, c}$, Guillermo Gonzálvez García ${ }^{2, d}$, \\ Luis Gerardo Castellanos',e
}

\footnotetext{
Unidad de Enfermedades Desatendidas, Tropicales y Transmitidas por Vectores, Departamento de Enfermedades Transmisibles y Análisis de Salud Organización Panamericana de la Salud. Washington DC, EE. UU.

Departamento de Enfermedades Transmisibles y Análisis de Salud, Organización Panamericana de la Salud. Lima, Perú.

a Licenciado en Ciencias, Máster en Ciencias, Especialista registrado en Salud Ambiental; b Doctor en Medicina, especialista en Salud Pública y Medicina Preventiva, Máster en Salud Pública; ‘ Bachiller en Administración de Empresas y Bachiller en Economía; d Doctor en Medicina, Máster en Salud Pública; e Doctor en Medicina, Doctorado en Epidemiología, Máster en Salud Pública.
}

Recibido: 26-04-14; Aprobado: 28-05-14

\begin{abstract}
RESUMEN
Las enfermedades infecciosas desatendidas (EID) afectan, principalmente, a las poblaciones que viven en condiciones aisladas y socioeconómicas de pobreza. Estas enfermedades, por su naturaleza crónica y silenciosa, aquejan con frecuencia a comunidades con voz política débil. Lo anterior se traduce en muy poca atención o prioridad política; lo que se refleja en mínimas e insuficientes acciones de prevención, vigilancia y control. Sin embargo, hay evidencia de que la situación está cambiando favorablemente en algunos países de las Américas. En los últimos años, varias resoluciones (acuerdos oficiales de los países miembros de la Organización Panamericana de la Salud/Organización Mundial de la Salud- OPS/ OMS), convenios regionales y mundiales en materia de salud pública, junto a un mayor compromiso por parte de la industria farmacéutica, y otros donantes y socios internacionales, en combinación con el desarrollo y uso de los planes de acción integrados, han permitido que los países intensifiquen las intervenciones públicas hacia el control de estas enfermedades y así alcanzar los objetivos de eliminación de las EID. La oncocercosis, la filariasis linfática, la enfermedad de Chagas, la lepra y la ceguera por tracoma, entre otras, han sido eliminadas en varios países o zonas endémicas, sin importar el nivel de desarrollo del país o área geográfica donde se localizan. La voluntad política reflejada en los recursos financieros suficientes en la próxima década, serán determinantes para lograr los objetivos regionales y nacionales de eliminación de las EID.
\end{abstract}

Palabras clave: Enfermedades infecciosas; Enfermedades tropicales desatendidas; Zoonosis; Malaria; Vigilancia epidemiológica; Políticas públicas de salud (fuente: DeCS BIREME).

\begin{abstract}
Neglected infectious diseases (NID) affect mainly isolated populations living in isolation and in poor socioeconomic conditions. These diseases, by their chronic and silent nature, often affect communities with a weak political voice. This translates into very little attention or political priority; which is reflected in minimal and insufficient preventive measures, monitoring and control. However, there is evidence this situation is changing favorably in some countries of the Americas. In recent years, several resolutions (official agreement of the member countries of the Pan American Health Organization / World Health Organization-PAHO / WHO), regional and global agreements on public health; with a greater commitment from the pharmaceutical industry, and other donors and international partners in combination with the development and use of integrated action plans have allowed countries to intensify public interventions to control these diseases and thus achieve target elimination of NID. Onchocerciasis, lymphatic filariasis, Chagas disease, leprosy and trachoma blindness, among others, have been eliminated in several countries and endemic areas, regardless of the level of development of the country or geographical area where they are located. The political decision reflected in adequate financial resources in the next decade will be crucial to achieving the goals of elimination of NID (regional and national).
\end{abstract}

Key words: Infectious diseases; Neglected tropical diseases; Zoonoses; Malaria; Epidemiological surveillance; Health public policy (source: MeSH NLM).

Citar como: Ault SK, Catalá Pascual L, Grados-Zavala ME, Gonzálvez García G, Castellanos LG. El camino a la eliminación: un panorama de las enfermedades infecciosas desatendidas en América Latina y el Caribe. Rev Peru Med Exp Salud Publica. 2014;31(2):319-25. 


\section{INTRODUCCIÓN}

Las enfermedades infecciosas desatendidas (EID) afectan a poblaciones vulnerables que viven en condiciones socioeconómicas de pobreza, y con frecuencia aisladas y con barreras para el acceso a los servicios de salud y educación. También ocurren entre individuos y familias con viviendas precarias y sin capacidad de satisfacer las necesidades de servicios básicos como agua saludable y saneamiento mejorado. Es frecuente, además, que estas poblaciones vulnerables vivan en las zonas semirrurales o cinturones de miseria de ciudades en países tropicales y subtropicales. Así también, las EID ocurren en comunidades que viven en zonas de conflicto o en condiciones ambientales deterioradas. Sin duda, las EID son una expresión y resultado de las condiciones de pobreza y marginación social y económica de ciertos grupos de población. Por compartir factores determinantes sociales y ambientales, estas enfermedades pueden presentar superposición geográfica, y es común encontrar personas con más de una EID al mismo tiempo. Por ejemplo, poliparasitismo por helmintos intestinales y protozoarios intestinales ${ }^{(1)}$, y diversos tipos de coinfección entre la enfermedad de Chagas y leishmaniosis ${ }^{(2)}$.

\section{CONTEXTO POLÍTICO}

Un hito en el compromiso político en salud pública de América Latina y el Caribe fue la aprobación de los estados miembros de la OPS/OMS de la resolución CD49.R19, el 9 de octubre de $2009^{(3)}$. En esta resolución se establecen las metas para el 2015 hacia el control de las helmintiasis transmitidas por el suelo, HTS o las geohelmintiasis (Ascaris lumbricoides, Trichuris trichiura, y las uncinarias - Necator americanus y Ancylostoma duodenale) y la esquistosomiasis (Schistosoma mansoni); así como las metas hacia la eliminación de más de diez de las EID y control de otras enfermedades relacionadas con la pobreza. Entre todas estas se incluye a: la enfermedad de Chagas; la oncocercosis, o ceguera de los ríos; la filariasis linfática (por Wuchereria bancrofti); la ceguera causada por tracoma; la lepra, o enfermedad de Hansen; la peste, e incluso la malaria; la rabia humana transmitida por perros; la sífilis congénita, y el tétano neonatal. La resolución CD49.R19 enfatiza la importancia de un abordaje integrado que incluya estrategias interprogramáticas con los programas de salud existentes, como el Programa Ampliado de Inmunizaciones (PAI); los programas de control de enfermedades transmitidas por vectores; los de Atención Integral de Enfermedades Prevalentes de la Infancia (AIEPI); los programas de escuelas saludables, etc. Sobre todo, acciones intersectoriales con educación, vivienda, desarrollo, agua y saneamiento básico, medioambiente, agricultura y ganadería, turismo e incluso el sector privado. En otras palabras, la resolución promueve todas las estrategias para un abordaje sostenible e integral de los factores sociales determinantes de la salud que perpetúan la transmisión de las EID. Esta resolución fue ratifica en el 2013 por la Organización de los Estados Americanos con la resolución AG/RES.2810(XLIII-O/13) (4).

Muchas son las expresiones formales de apoyo y acuerdos oficiales que los países han comprometido por esta causa. En el 2010, el Consejo Directivo de la OPS aprobó la resolución CD50.R17 ${ }^{(5)}$, para la prevención de enfermedad de Chagas. En el 2011, también se formalizó la resolución CD51.R9, una estrategia y plan de acción sobre la malaria. Al nivel global, la OMS publicó en 2012 la "hoja de ruta" (6) sobre enfermedades tropicales desatendidas (ETD). Su propósito es orientar la implementación de las políticas y estrategias para superar el impacto global de las enfermedades tropicales desatendidas. Además, en el 2012 se firmó la "Declaración de Londres sobre las ETD" (7), en la que distintas organizaciones (públicas y privadas), y gobiernos se comprometieron a eliminar diez de esas enfermedades para el año 2020. Entre los acuerdos que se alcanzaron cabe resaltar que varias compañías farmacéuticas garantizaron la donación de medicamentos para combatir varias EID. Una vez más, en el 2012, la Asamblea Mundial de la Salud (AMS) adoptó la resolución WHA65.21 ${ }^{(8)}$, que hace un llamado a la acción mundial para la eliminación de la esquistosomiasis. En el 2013, se aprobó también la resolución WHA66.12 ${ }^{(9)}$, que hace otro llamado mundial para eliminar o controlar 17 enfermedades tropicales desatendidas con metas establecidas para el año 2015 y para el 2020.

Basado en lo anterior, se puede decir que se tiene el compromiso político nacional e internacional, y que se dispone de intervenciones de salud pública costoefectivas, para controlar y eliminar varias EID. Por lo tanto, urge aunar esfuerzos, incluyendo los de carácter interprogramático e intersectorial, para combatir las EID y contribuir al logro de los Objetivos de Desarrollo del Milenio ${ }^{(10)}$, y su sostenibilidad pos-2015.

\section{SITUACIÓN ACTUAL Y PROGRESO EN LAS AMÉRICAS EN EL COMBATE A LAS ENFERMEDADES INFECCIOSAS DESATENDIDAS}

En las Américas, la OMS verificó la eliminación de la oncocercosis en Colombia en el 2013, y se prevé que Ecuador también lo logre entre el 2014 y 2015. México y Guatemala están en vigilancia epidemiológica postratamiento y, si las evaluaciones del 2014 son favorables, ambos 
podrían solicitar la verificación de la eliminación en 2015. Posterior a esto, solo dos focos colindantes de transmisión localizados en el área amazónica, Yanomami entre Brasil y Venezuela, serían el desafío para que el continente entero esté libre de esta enfermedad. En cuanto a la ceguera por tracoma, se estima que 50 millones de personas viven aún en zonas de riesgo en cuatro países. En Brasil existen focos endémicos activos por todo el país. Guatemala, con un solo foco detectado, evaluará en el 2014 si el tracoma dejó de ser un problema de salud pública. En Colombia, en 2010, se evidenció por primera vez tracoma en comunidades indígenas. Para prevenir la ceguera por tracoma, la OPS/OMS recomienda la estrategia SAFE, que promueve la mejora del saneamiento, y las condiciones ambientales, la higiene facial con agua limpia, para prevenir el tracoma folicular y la cirugía en los casos de triquiasis tracomatosa o entropión.

En el 2011, tres países fueron eliminados de la lista de la OMS de países endémicos para filariasis linfática (Costa Rica, Surinam y Trinidad y Tobago), después de años de esfuerzo en localizar y tratar personas infectadas, mejorando los servicios de agua potable, saneamiento básico y drenaje. Actualmente existen cuatro países endémicos (Brasil, Haití, Guyana y República Dominicana), que están realizando esfuerzos para reducir la transmisión de la enfermedad y avanzar hacia su eliminación. Una de las intervenciones, común en estos cuatro países, es la administración masiva de medicamentos (citrato de dietilcarbamazina + albendazol) a la población en riesgo de infección, y promover la mejora del diagnóstico y la atención de las personas con morbilidad por la infección.

La enfermedad de Chagas es endémica en 21 países de las Américas, afecta entre 6 y 8 millones de personas. El número estimado de casos nuevos de Chagas se ha reducido en $32 \%$ pasando de 41000 casos en el año

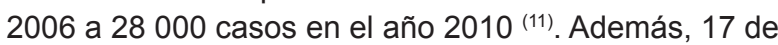
estos países han eliminado la transmisión de la enfermedad por el principal vector intradomiciliar a nivel nacional o subnacional, y 20 de los 21 países endémicos han establecido el tamizaje de T. cruzi en el $100 \%$ de los bancos de sangre (datos del programa regional EID/ OPS al 2014). Las iniciativas intergubernamentales de cooperación, formadas por los países endémicos, junto al apoyo de socios y aliados como JICA de Japón y la propia OPS, han sido clave para alcanzar estos logros. Pero todavía queda el reto de eliminar la transmisión materno-infantil y los brotes de la enfermedad causados por consumo de alimentos y bebidas.

En el 2012, se informó a OPS/OMS que 24,8 millones de niños entre 1 y 15 años fueron desparasitados con albendazol o mebendazol en la región de las Américas ${ }^{(12)}$; y se estimó para ese mismo año 49,3 millones de niños en riesgo de infección por geohelmintiasis. Actualmente, 18 países en las Américas elaboraron sus propuestas iniciales de planes de acción hacia el control de geohelmintiasis, y enfatizaron la importancia de integrar la mejora del acceso a agua segura, saneamiento mejorado, y educación en higiene ${ }^{(13)}$. Adicionalmente, cuatro países (Honduras, Brasil, Guatemala y Colombia) han lanzado planes integrados de acción hacia el control y la eliminación de varias EID, y otros países están elaborando sus planes de acción. También se han elaborado planes o estrategias específicas para la eliminación de ciertas EID en la región (por ejemplo para oncocercosis), y subregiones (por ejemplo, para combatir conjuntamente la filariasis linfática y la malaria en la isla La Española, y la enfermedad de Chagas en Centroamérica).

En lasAméricas, un promedio 58000 casos de leishmaniosis cutánea y mucocutánea se notifican anualmente. De estos, cerca del $75 \%$ de los casos de leishmaniosis cutánea se registran en diez países de la región, Brasil notifica alrededor del 30\% de esto. Sin embargo, las tasas más altas se observan en Panamá y Nicaragua. Además, un promedio de 4000 casos de leishmaniosis visceral se reportan cada año en la región, con una tasa de letalidad del $7 \%$. Además, existe coinfección de leishmaniosis con el VIH, lo que complica el manejo clínico de los casos. La OPS proporciona cooperación técnica directa y facilita la capacitación en la vigilancia, la prevención, el diagnóstico de laboratorio, el tratamiento y el control de la enfermedad. Recientemente, la OPS/OMS concluyó la elaboración de un curso a distancia para divulgar el diagnóstico y tratamiento de la enfermedad ${ }^{(14)}$, y publicó una guía que compila la evidencia y las recomendaciones para el tratamiento de las leishmaniosis en las Américas ${ }^{(15)}$.

\section{UNA PERSPECTIVA DE LAS ENFERMEDADES INFECCIOSAS DESATENDIDAS EN PERÚ}

Perú es un país endémico para varias EID que afectan, principalmente, a población que vive en condiciones de pobrezaymarginalización. Estasenfermedadesincluyen algunas que son prevenibles con la administración masiva de medicamentos (AMM) en función de la prevalencias ${ }^{(16)}$, según recomienda la OMS. Entre estas se pueden mencionar: las helmintiasis transmitidas por el suelo y la fascioliasis. También existen muchas EID no-prevenibles (con la AMM), pero sí endémicas en el Perú. entre ellas las leishmaniosis (cutánea, mucocutánea), la peste, la cisticercosis, la lepra, la equinococosis, la enfermedad de Chagas, la úlcera de Buruli, la enfermedad de Carrión y las mordeduras por las arañas de genus, Loxoceles principalmente; algunas de estas enfermedades se tratan en detalle en esta edición especial de la revista. 
Desde la perspectiva del programa regional de EID de la OPS/OMS, se discutirán principalmente las EID en el Perú que son susceptibles de ser controladas con AMM, y la ejecución de otras estrategias intersectoriales para el logro de las metas que incluyen el sector de la salud pública veterinaria, el manejo integrado de vectores, el manejo individual de pacientes, el acceso a agua segura, el saneamiento básico y las buenas prácticas de higiene y la manipulación de alimentos. También se discutirán brevemente los logros recientes en el combate a la enfermedad de Chagas.

\section{LAS HELMINTIASIS TRANSMITIDAS POR EL SUELO EN PERÚ}

Los principales helmintos transmitidos por contacto con el suelo, son infecciones que afectan principalmente el desarrollo físico y cognitivo de los niños ${ }^{(17)}$, debido a la malabsorción de micronutrientes que puede desencadenar anemia, reducción de la capacidad de ingesta de alimentos, obstrucción de las vías intestinales y biliares (Ascaris), prolapso rectal (trichuriasis), y anemia por deficiencia de hierro debido a la infección por uncinarias. La infección por HTS disminuye la capacidad de trabajo de los adultos e incrementa el ausentismo escolar entre los niños, repercutiendo negativamente en el desarrollo social y económico de las comunidades con altas prevalencias de geohelmintos ${ }^{(18,19)}$.

En Perú, de acuerdo al algoritmo de la OMS (20), se estima que 3 millones de niños entre 1 y 15 años están en riesgo de infección por HTS, por falta de acceso a saneamiento mejorado (Tabla 1). En una revisión sobre prevalencia e intensidad de infección por geohelmintos en Latinoamérica y el Caribe se señala que la prevalencia de esta infección en los municipios encuestados de Perú (años 2000-2010) puede oscilar entre menos 1\% hasta $77,9 \%{ }^{(21)}$. Por lo tanto, el país debe documentar mejor (realizar un mapeo más preciso), la prevalencia actual e intensidad de infección a nivel nacional. Perú desparasita individualmente a los niños diagnosticados con infección por HTS en los centros de atención primaria de la red pública y en algunas organizaciones no gubernamentales, que están desparasitando colectivamente aunque no se dispone de información exacta y sistemática anual de cuantos niños se están desparasitando cada año (Tabla 1). Para disponer de esta información, la OPS/OMS recomienda que el Ministerio de Salud, en colaboración con los ministerios de Educación, Desarrollo e Inclusión Social, coordine las actividades de desparasitación y control de las geohelmintiasis, en colaboración adicional de las ONG y otros aliados.

\section{FASCIOLIASIS EN PERÚ}

Otra EID prevenible con la administración masiva de medicamentos en el Perú es la fascioliasis. Esta infección es causada por un trematodo del género Fasciola ( $F$. hepática y $F$. gigantica), que afecta principalmente el hígado. Esta enfermedad pertenece al grupo de infecciones transmitidas por alimentos y se considera una zoonosis. En el altiplano de Perú y Bolivia, se dan las condiciones sociales y económicas ideales para la transmisión intensa de la fascioliasis, al punto de convertirla en un problema de salud pública, por la alta prevalencia de infección en humanos, se estima que decenas de miles de personas están infectadas. Muchas

Tabla 1. Población de preescolares y escolares en riesgo de infección por geohelmintos, niños desparasitados reportados y cobertura nacional, Perú, 2005-2011

\begin{tabular}{|c|c|c|c|c|c|c|c|c|}
\hline \multirow[b]{2}{*}{ Año } & \multicolumn{4}{|c|}{ Preescolares } & \multicolumn{4}{|c|}{ Escolares } \\
\hline & $\begin{array}{l}\text { Población estimada } \\
\text { que requiere trata- } \\
\text { miento quimiopre- } \\
\text { ventivo }\end{array}$ & $\begin{array}{l}\text { Número de ni- } \\
\text { nuos reportados } \\
\text { como despara- } \\
\text { sitados }\end{array}$ & $\begin{array}{l}\text { Medicamento } \\
\text { utilizado }\end{array}$ & $\begin{array}{c}\text { Cobertura } \\
\text { nacional }\end{array}$ & $\begin{array}{l}\text { Población esti- } \\
\text { mada que requie- } \\
\text { re tratamiento } \\
\text { quimiopreventivo }\end{array}$ & $\begin{array}{l}\text { Número niños } \\
\text { reportados } \\
\text { como despa- } \\
\text { rasitados }\end{array}$ & $\begin{array}{l}\text { Medicamento } \\
\text { utilizado }\end{array}$ & $\begin{array}{c}\text { Cobertura } \\
\text { nacional }\end{array}$ \\
\hline 2005 & 2380221 & - & - & - & 5910262 & 1539500 & alb & $26,1 \%$ \\
\hline 2006 & 2377458 & - & - & - & 5905157 & 1729412 & alb/mbd & $29,3 \%$ \\
\hline 2007 & 2375553 & - & - & - & 5896390 & 109900 & alb & $1,9 \%$ \\
\hline 2008 & 2373462 & 3500 & alb/mbd & $0,2 \%$ & 5885700 & - & - & - \\
\hline 2009 & 999228 & 3632 & alb/mbd & $0,4 \%$ & 2477333 & 2217512 & alb/mbd & $89,5 \%$ \\
\hline 2010 & 996559 & 105542 & alb/mbd & $10,6 \%$ & 2473590 & 278906 & alb/mbd & $11,3 \%$ \\
\hline 2011 & 992649 & - & - & - & 2470914 & - & - & - \\
\hline 2012 & 858942 & - & - & - & 2144562 & - & - & - \\
\hline
\end{tabular}

alb: albendazol, mbd: mebendazol

Fuente: World Health Organization. Preventive Chemotherapy (PC) databank. Disponible en: http://www.who.int/neglected_diseases/preventive_chemotherapy/sth/en/ 
de estas infecciones ocurren también por el consumo de vegetales contaminados. Además, el impacto negativo de la fascioliasis en la economía ganadera no es menor de US\$ 50 millones por año ${ }^{(22)}$.

En el Perú y en Bolivia se concluyó que el diagnóstico y tratamiento individual no es siempre la forma más costoefectiva de combatir la enfermedad y, en su lugar, se hicieron estudios piloto, para tratar una vez al año con AMM a todos los niños en edad escolar, que viven en las zonas endémicas. Desde el año 2008, ambos países han implementado intervenciones de administración masiva de triclabendazol para combatir la fascioliasis (Tabla 2). Pero para controlar mejor la transmisión, no es suficiente administrar solo el medicamento; también es necesario abordar otros aspectos intersectoriales que incluyen la salud veterinaria, el control vectorial, facilitar el acceso a agua segura y saneamiento básico, promover la educación en salud e higiene, particularmente para cambiar ciertos comportamientos relacionados con la costumbre de comer plantas acuáticas crudas (berros) que perpetúan la transmisión de la infección. En el Perú se han emprendido varias acciones en el marco político y técnico, así como en la movilización de recursos para intentar superar los retos de control de estas infecciones.

En el marco político, un punto para destacar es que el Gobierno del Perú, en su compromiso de combatir integradamente las EID y otras enfermedades relacionadas con la pobreza, firmó un convenio con la OPS para mejorar el acceso a agua y saneamiento básico facilitando la conformación del Equipo Técnico Regional Agua y Saneamiento (ETRAS). El establecimiento de este acuerdo coincide con algunas de las recomendaciones de un taller sobre geohelmintiasis que se realizó en el $2013^{(13)}$, en el que se resaltó que los países deben avanzar hacia el control de los HTS, reforzando la colaboración interprogramática e intersectorial y mejorar la capacidad técnica con el apoyo del equipo de ETRAS y otras unidades técnicas de la OPS. Esta es una forma en la que OPS y el ETRAS contribuirán a fortalecer el abordaje de las EID tomando en consideración los determinantes sociales de la salud, y con la mirada en el manejo ambiental con atención a los riesgos provocados por el cambio climático.

Tabla 2. Personas tratadas por fascioliasis humana con triclabendazol, del 2008 al 2012 en Perú y Bolivia

\begin{tabular}{lccccc}
\hline País & $\mathbf{2 0 0 8}$ & $\mathbf{2 0 0 9}$ & $\mathbf{2 0 1 0}$ & $\mathbf{2 0 1 1}$ & $\mathbf{2 0 1 2}$ \\
\hline Perú & \multicolumn{2}{c}{36601} & 49063 & 2176 \\
\hline Bolivia* & 71456 & 223946 & 106936 & 134782 \\
\hline
\end{tabular}

*La segunda y tercera campaña de administración masiva de medicamentos en Bolivia se realizó a lo largo de dos años (2009-2010 y 20102011 respectivamente)
En el marco técnico, el país ha participado en varios talleres regionales organizados por la OPS/OMS y otras entidades, mostrando su interés en desarrollar un plan estratégico y acciones integradas hacia el control y la eliminación de las EID. Adicionalmente, el Perú y Bolivia ${ }^{(23)}$, han sido pioneros en generar la evidencia de la efectividad de la administración masiva con triclabendazol, junto con otras intervenciones intersectoriales integradas para combatir la infección por fascioliasis.

En el 2012, Perú, apoyado el Departamento de Relaciones Exteriores, Comercio y Desarrollo Canadá (CIDA-Canadá), y la OPS, estableció la línea de base de prevalencia de HTS en dos municipios, (Bagua y Condorcanqui), en la región del Amazonas, fortaleciendo las capacidades de los equipos de salud de la Dirección Regional de Salud del Amazonas para mejorar el control de las geohelmintiasis. Entre las lecciones aprendidas, se destaca la necesidad de involucrar a diferentes actores desde la planificación de las estrategias de control, la importancia de la participación de las comunidades indígenas en las intervenciones y el papel clave de la apropiación de la información por parte de las autoridades regionales y locales para darle sostenibilidad a las acciones. Esta experiencia puede ser de gran ayuda para expandir e institucionalizar a nivel nacional estas actividades hacia el control de las geohelmintiasis en el Perú. En el marco de movilización de recursos, en enero del 2014, un proyecto presentando por una ONG trabajando en el país, y sus colaboradores a una convocatoria de subvenciones del END7 Fund, fue aprobado para sistematizar la actividades de desparasitación y el monitoreo y la evaluación de un programa de control de HTS en el departamento de Ucayali. Además, durante el taller regional ya mencionado, representantes del Ministerio de Salud, Ministerio de Educación y el Ministerio de Desarrollo e Inclusión Social prepararon un borrador de un proyecto piloto para la provincia de Bagua, departamento de Amazonas, hacia el fortalecimiento de los lazos intersectoriales para el control de los HTS en el Perú y sistematizar la experiencia piloto.

\section{LA ENFERMEDAD DE CHAGAS EN PERÚ}

Con apoyo de la Agencia Canadiense de Cooperación Internacional (CIDA-Canadá) el país logró la eliminación de la transmisión vectorial de la enfermedad de Chagas en Tacna (diciembre de 2009) y Moquegua (septiembre de 2011) y se lograron avances en Arequipa donde aún se estima que el $44 \%$ de los distritos deben ser intervenidos con rociado residual intra y peridomiciliar para combatir el vector. Actualmente, se ha iniciado por el Ministerio de Salud una encuesta seroepidemiológica en menores de 5 años en los departamentos de la macrorregión sur del 
Perú lo que permitirá conocer con mayor precisión las localidades donde aún persiste la transmisión vectorial $y$, de esta manera, focalizar las intervenciones.

\section{REFLEXIONES SOBRE EL CAMINO A SEGUIR}

Para lograr y mantener los logros en el control y la eliminación de las EID en las América, hay que destacar algunos puntos clave.

El apoyo gubernamental a los programas de control y eliminación de las EID, debe reflejarse en un presupuesto asignado a dichos programas y a otros sectores gubernamentales pertinentes. Aunque la cooperación técnica de la OPS ha sido importante al obtener capital generador de inversiones, para promover intervenciones integradas, la movilización de los recursos por parte del gobierno, y con el apoyo adicional de otros socios estratégicos seguirá siendo esencial para alcanzar los objetivos y éxitos en salud pública.

Las autoridades de los países, a través de su legislación, deben facilitar los procedimientos para la donación, la importación y el acceso a los medicamentos para diagnosticar y tratar las EID, así como mejorar el manejo de casos basados en el mejor conocimiento científico disponible. En los países que comparten poblaciones de trabajo con flujos migratorios, la vigilancia y la educación asumidas de manera colaborativa, facilitará los esfuerzos de eliminación de enfermedades como ceguera por tracoma, filariasis linfática, oncocercosis y malaria. En las áreas en riesgo de EID, las autoridades también pueden considerar el abordaje de los determinantes sociales y ambientales de la salud, incluyendo facilitar el acceso a agua segura, saneamiento básico, drenaje, educación en higiene, vivienda y control integrado de vectores. Se ha observado que hay mayor impacto si los programas sociales de suplementos de alimentos para escolares, cuando al mismo tiempo se mejora el acceso y calidad del agua y el saneamiento en las escuelas, junto con la administración de antihelmínticos.

Los avances hacia el control y la eliminación de las EID son más eficientes cuando las autoridades de un país elaboran e implementan programas, políticas y planes intersectoriales integrados en colaboración con socios y aliados clave. Además, con los procesos de reforma del sector salud y la Cobertura Universal en Salud (incluida la atención primaria de la salud renovada), que actualmente se está expandiendo en la región, más comunidades tendrán acceso a servicios de prevención y tratamiento para estas y otras enfermedades. La utilización de directrices regionales y otros instrumentos técnicos, junto con la cooperación técnica prestada por la OPS/OMS, facilitará los esfuerzos de los países para alcanzar la eliminación de las EID.

\section{COMENTARIOS FINALES}

Es importante recordar que las Américas tiene una historia de éxito en la eliminación de varias enfermedades prevenibles mediante la vacunación, entre ella a la viruela, la poliomielitis y el sarampión. Las Américas también lideran la eliminación de varias EID utilizando efectivamente la administración de medicamentos, el manejo intensivo de los casos, y mejorando las condiciones sanitarias y ambientales. Como resultado de estos esfuerzos integrados, tres países de la región (Costa Rica, Surinam, Trinidad y Tobago) ya no se consideran más como países endémicos para la filariasis linfática; asimismo, se ha conseguido alcanzar la eliminación de la transmisión de la enfermedad de Chagas, por el principal vector domiciliario y por transmisión sanguínea, en varios países y subregiones, así como también, la eliminación de lepra como problema de salud pública a primer nivel administrativo nacional en 24 países endémicos en la región, (18 de estos también en segundo nivel administrativo). Además, con verificación por la OMS, de la eliminación de la oncocercosis en Colombia en el 2013 y el proceso que sigue Ecuador durante el 2014 para ser el segundo país del mundo en alcanzar este logro.

A través de la expansión de las acciones de vigilancia, control y eliminación de las EID, Perú y los demás países endémicos de las Américas tienen una gran oportunidad de fortalecer la salud pública, contribuir directamente a los objetivos económicos y políticos de sus gobiernos, y responder de una manera más integral a las comunidades más necesitadas y afectadas por las EID. El abordaje integral de control y eliminación de las EID en América Latina y el Caribe es un imperativo ético para alcanzar la justicia y la equidad social para la región de las Américas.

Contribuciones de autoría: SKA ha organizado la concepción y diseño del artículo, la recolección de resultados, el análisis e interpretación de datos, la redacción, y revisión crítica. Además, MEGZ realizo la recolección de resultados y redacción del artículo. LCP, LGC y GGG realizaron el análisis e interpretación de datos y revisión crítica del artículo. SKA y LGC aprobaron la versión final.

\section{Fuentes de financiamiento: OPS/OMS}

Conflictos de interés: Los autores declaran no tener conflictos de interés. 


\section{REFERENCIAS BIBLIOGRÁFICAS}

1. Salcedo-Cifuentes M, Florez O, Bermúdez A, Hernández L, Araujo C, Bolaños, MV. Intestinal parasitism prevalence amongst children from six indigenous communities residing in Cali, Colombia. Rev Salud Publica (Bogota). 2012 Jan-Feb;14(1):156-68.

2. Perez CJ, Lymbery AJ, Thompson RC. Chagas disease: the challenge of polyparasitism?. Trends Parasitol. 2014 Apr;30(4):176-82. doi: 10.1016/j. pt.2014.01.008.

3. Organización Panamericana de la Salud. Resolución CD49.R19 del Consejo Directivo de OPS sobre la eliminación de las enfermedades desatendidas y otras infecciones relacionadas con la pobreza. Washington, DC: OPS; 2009.

4. Organización de los Estados Americanos. Resolución AG/RES.2810 (XLIII-O13): Eliminación de las enfermedades desatendidas y otras infecciones relacionadas con la pobreza. La Antigua, Guatemala. 6 de junio de 2013.

5. Organización Panamericana de la Salud. Resolución CD50.R17: Estrategia y plan de acción para la prevención, el control y la atención de la enfermedad de Chagas. Washington, D.C., EUA, del 27 de septiembre al 1 de octubre del 2010.

6. World Health Organization. Accelerating work to overcome the global impact of neglected tropical diseases. A road map for implementation. Washington, DC: WHO; 2012.

7. World Health Organization. Uniting to Combat Neglected Tropical Diseases. London Declaration on Neglected Tropical Diseases [Internet]. Washington, DC: WHO; [citado el 5 de febrero del 2014]. Disponible en: http://www. who.int/neglected_diseases/London_ Declaration_NTDs.pdf

8. Organización Mundial de la Salud. Resolución WHA65.12: Eliminación de la esquistosomiasis. Geneva, Switzerland. 26 de mayo de 2012.

9. World Health Organization. Resolución WHA66.12: Neglected tropical diseases. Washington, D.C., EUA, 27 May 2013.
10. Holveck JC, Ehrenberg JP, Ault SK, Rojas R, Vásquez J, Cerqueira MT, et al. Prevention, control, and elimination of neglected diseases in the Americas: Pathways to integrated, interprogrammatic, intersectorial action for health and development. BMC Public Health. 2007 Jan 17;7:6.

11. World Health Organization. Sixtyfifth World Health Assembly. Provisional agenda item 13.16. Progress reports [Internet]. Washington, DC: WHO; [citado el 5 de febrero del 2014]. Disponible en: http://apps.who.int/gb/ ebwha/pdf_files/WHA65/A65_26en.pdf

12. World Health Organization. Neglected tropical diseases: PCT databank [Internet]. Washington, DC: WHO; [citado el 5 de febrero del 2014]. Disponible en: http://www.who.int/neglected_diseases/preventive_chemotherapy/sth/ en/

13. Organización Panamericana de las Salud. Taller de capacitación en las pautas operativas para la puesta en marcha de actividades integradas de desparasitación para las geohelmintiasis [Internet]. Bogotá: OPS; 2013 [citado el 5 de febrero del 2014]. Disponible en: http:// www.paho.org/hq/index.php?\%20 option $=$ com_docman $\&$ task $=$ doc view\&gid=23556\&Itemid=

14. Organización Panamericana de la Salud. Campus Virtual de Salud Pública. Curso de autoaprendizaje de leishmaniosis en las Américas: diagnóstico y tratamiento [Internet]. Washington, DC: WHO; [citado el 5 de febrero del 2014]. Disponible en: http://cursos. campusvirtualsp.org/login/index.php

15. Organización Panamericana de la Salud. Leishmaniasis en las Américas. Recomendaciones para el tratamiento. Washington, DC: OPS; 2013.

16. World Health Organization. Helminth control in school-age children: a guide for managers of control programmes. 2nd ed. Geneva: WHO; 2011.

17. Oberhelman RA, Guerrero ES, Fernández ML, Silio M, Mercado D, Comiskey $\mathrm{N}$, et al. Correlations between intestinal parasitosis, physical growth, and psychomotor development among infants and children from rural Nicaragua. Am J Trop Med Hyg. 1998 Apr;58(4):470-5.

18. Guyatt H. Do intestinal nematodes affect productivity in adulthood? Parasitol Today. 2000 Apr;16(4):153-8.

19. World Health Organization. Working to overcome the global impact of neglected tropical diseases: First WHO report on neglected tropical diseases. Geneva: WHO; 2010.

20. Soil-transmitted helminthiases: estimates of the number of children needing preventive chemotherapy and number treated, 2009. Wkly Epidemiol Rec. 2011 Jun 17;86(25):257-67.

21. Pan American Health Organization. Prevalence and intensity of infection of soil-transmitted helminths in Latin America and the Caribbean Countries: Mapping at second administrative level 2000-2010. Washington, DC: PAHO; 2011.

22. Espinoza JR, Terashima A, HerreraVelit P, Marcos LA. Fascioliasis humana y animal en el Peru: impacto en la economía de las xonas endémicas. Rev Peru Med Exp Salud Publica. 2010 Oct-Dec;27(4):604-12.

23. Villegas F, Angles R, Barrientos R, Barrios G, Valero MA, Hamed K, et al. Administration of triclabendazole is safe and effective in controlling fascioliasis in an endemic community of the Bolivian Altiplano. PLoS Negl Trop Dis. 2012;6(8):e1720. doi: 10.1371/ journal.pntd.0001720.

Correspondencia: Steven Kenyon Ault

Dirección: Regional Program for Neglected Infectious Disease. Department of Communicable Disease and Health Analysis. Pan American Health Organization/WHO. $52523^{\text {rd }}$ Street NW. Washington DC. EE. UU. Teléfono: +2029743896

Correo electrónico:aultstev@paho.org 